\title{
A new classification system of nasal contractures
}

\author{
Geunuck Chang', Donghak Jung² \\ 'Hanabi Rhinoplasty Clinic, Seoul, Rep. of Korea, ${ }^{2}$ Shimmian Rhinoplasty Clinic, Seoul, Rep. of Korea
}

Background: The nasal contracture is one of the most severe complications that follow rhinoplasty. The proposed reasons for this complication are a capsular contraction from the silicone implant, pressure necrosis of the lower lateral cartilage due to the long nasal implant, and inflammation after alloplastic implantation. The severity and extent of nasal contracture vary depending on the cause and duration. Treatment of nasal contractures is very challenging and different from normal rhinoplasty procedures. To the best of our knowledge, an established classification has yet to be introduced for effective treatment.

Objective: To introduce a system for the classification of nasal contractures, for effective treatment decision-making.

Methods: Data from 245 nasal contractures were reviewed retrospectively. Pre-operatively, a complete rhinological examination including photographs was performed and a prior surgical history was taken. Two hundred forty-five patients had surgical treatments with various procedures. The authors classified the 245 cases according to severity and analyzed the patients' data, including previous implants, infection history, and surgical procedures.

Results: Nasal contractures were classified into 6 categories. Type II (symmetric type, 42.4\%) and Type III (asymmetric type, 33.5\%) were the most common. A previous silicone implant was found in $77 \%$ of all cases of nasal contractures. A previous infection occurred in $56 \%$ of the cases. Extended spreader graft and strut graft were used for surgical treatment in $87 \%$ of the cases.

Conclusion: The authors have presented a new classification system of nasal contractures. Histories of a previous infection and previous silicone implant could be relevant parameters, however, it is not clear if they are direct causative factors. There was no significant correlation between the surgical management and type of nasal contracture.

Level of evidence: 3

Keywords: rhinoplasty; nasal contracture; classification; extended spreader graft; strut graft; alar retraction

\section{Introduction}

The nasal contracture is one of the most severe complications that follow a rhinoplasty. The nasal contracture causes the distortion such as nasal shortening or asymmetry and its surgical treatment is also more difficult than normal rhinoplasty and the most challenging aspect. The cause of nasal contractures has not been elucidated, but repeated surgery and inflammation are considered to be the predisposing factors. Other possible factors include a capsular contraction from the silicone implant, pressure necrosis of the lower lateral cartilage resulting from the long nasal implant, trauma, and an immunological mechanism $[1,2]$. The nasal contracture causes changes in the soft tissue, as well as in the supportive structures of the nose, resulting in deformity such as the shortening or asymmetry of the nose and retraction of part of the soft tissue [2]. Specifically, the nasal tip is supported only by cartilage, so deformity of the nose by the contracture is relatively more severe than the nasal bridge. The nasal contracture causes various forms and degrees of deformity depending on the cause, the site of tissue damage, 
and the period of injury. Different treatment methods should be applied depending on each condition. Since there has yet to be clear classification criteria for nasal contractures, there have been difficulties in classifying patients, establishing treatment plans, or reporting treatment outcomes to the academic world. The purpose of this study is to present a new classification standard for nasal contractures and to provide a unified standard for research and the classification and treatment of patients.

\section{Materials and methods}

From June 2011 to June 2015, the study was carried out on

Table 1. patient analysis

\begin{tabular}{|lc|}
\hline 2011.6-2015.6 & 245 patients \\
\hline Age & $37.3 \pm 12.1(20-69)$ \\
\hline M:F & $36: 209$ \\
\hline Previous infection & $137 / 245(56 \%)$ \\
\hline First Op. (year ago) & $9.3 \pm 8.6$ \\
\hline Last Op. (year ago) & $3.4 \pm 5.8$ \\
\hline
\end{tabular}

245 patients who underwent surgery for nasal contracture. Before surgery, the prior surgical history (previous surgeries and the period until reoperation, types of implants used in prior surgeries, and infection history) was examined and a rhinological examination, including photographs, was conducted. Each patient received surgical treatment using a variety of surgical techniques and was retrospectively analyzed through a chart review after surgery.

\section{Results}

The age of the patients ranged from 20 to 69 years old with an

Table 2. Classification of nasal contracture

\begin{tabular}{ll}
\hline Type I & Partial contraction \\
\hline Type II & Symmetric contraction \\
\hline Type III & Asymmetric contraction \\
\hline Type IV & General contraction \\
\hline Type V & Contraction with skin deformity \\
\hline Type VI & Destructive contraction \\
\hline
\end{tabular}
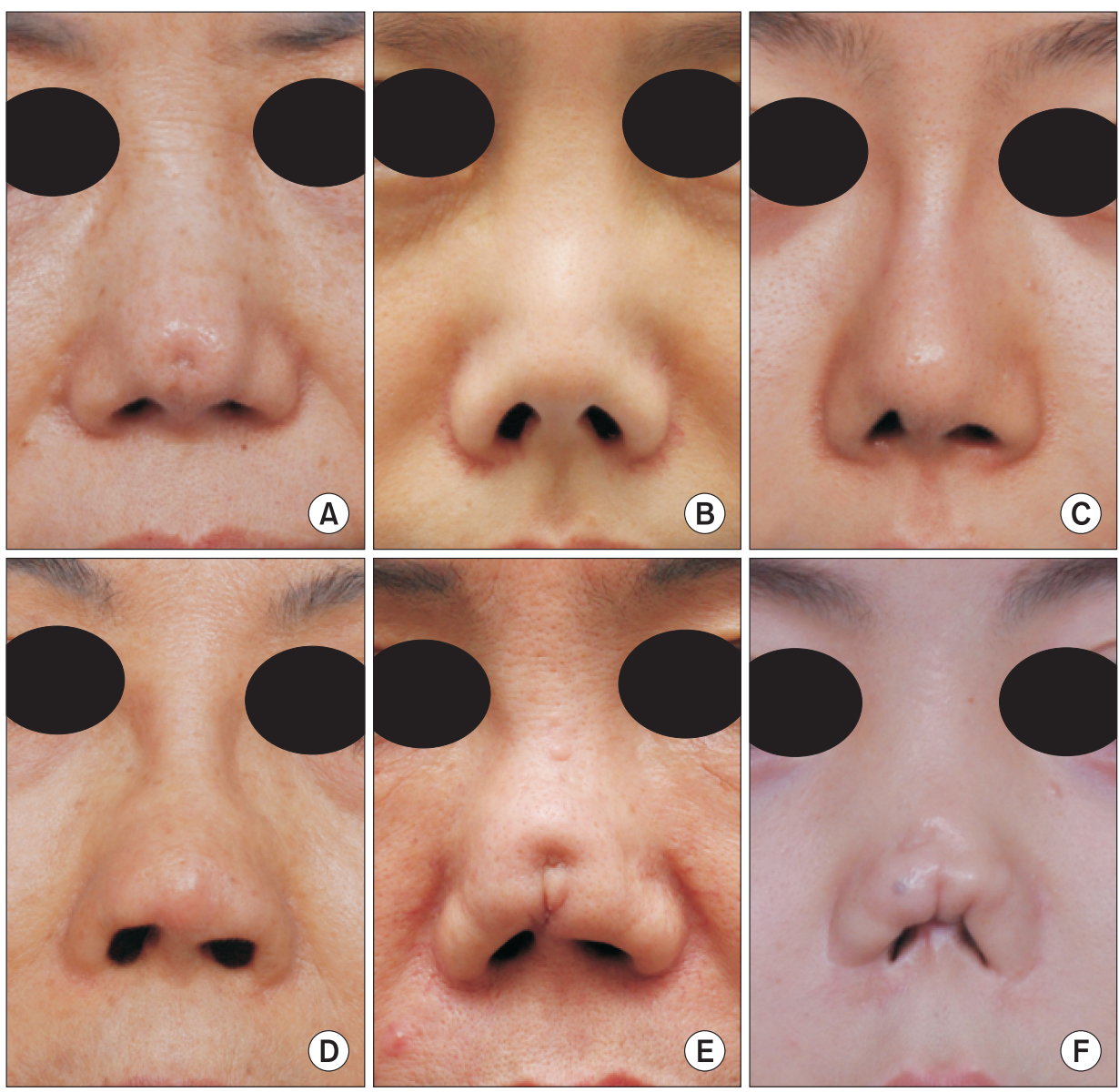

Fig. 1. Classification of nasal constracure. (A) Type I: partial contracture, (B) Type II: symmetric contracture, (C) Type III: asymmetric contracture, (D) Type IV: general contracture, (E) Type V: contracture with skin deformity, (F) Type VI: destructive contracture. 
average age of $37.3 \pm 12.1$. The male to female ratio was $36: 209$, with an overwhelming majority of women. The period from the initial surgery to the surgery at our clinic was $9.3 \pm 8.6$ years. On average from the amount of time between the patient's last surgery to surgery at our clinic was $3.4 \pm 5.8$ years (Table 1 ).

Two hundred forty-five nasal contractures were classified into 6 types according to the deformed nasal shape and its severity; Type I is a partial contracture that shows a partial retraction of the skin. Type II is a symmetric contracture that shows a shortening of the nose tip, while the symmetry of the nose is maintained. Type III is an asymmetric contracture and shortening. Type IV is a general contracture that causes an overall deformity across the nasal tip and the nasal bridge. Type $V$ involves deformity of the skin accompanied by a severe external skin defect with cartilage damage that requires external skin excision or minor flap surgery. Type VI is a destructive contracture accompanied by the loss of the normal structures of the nose that requires nasal reconstruction (Table 2, Fig. 1).

Two hundred forty-five cases were classified into 6 types. Type II (symmetric type, $42.4 \%$ ) was the most common, followed by type III (asymmetric type, 33.5\%) (Fig. 2).

The silicone implant was the most common (77\%) implant used in a previous surgery. The results of the analysis of each type showed that the silicone implant was the most common

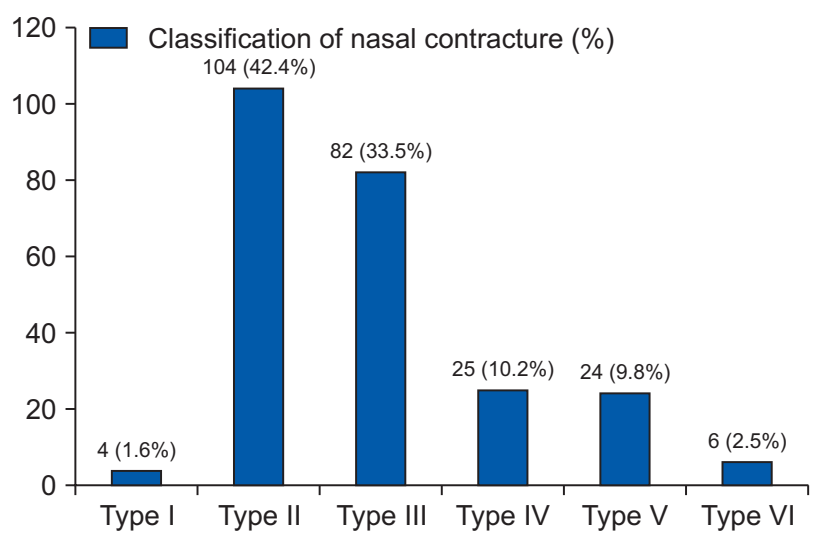

Fig. 2. Classification of nasal contracture. material used in previous surgeries, followed by e-PTFE (9\%) and costal cartilage (6\%) (Fig. 3, Table 3).

Of all the patients, one hundred thirty-seven patients (56\%) had a previous infection history associated with surgery after the previous surgery (Table 4 ).

Each patient underwent surgery by the authors for the treatment of nasal contracture. For the surgical procedures used, an extended spreader graft (ESG) and strut graft using costal cartilage was used to extend the nasal tip in $87 \%$ (213 cases) and septal reconstruction was carried out to improve the supporting force of the septum in $10 \%$ (25 cases). The patients of approximately $3 \%$ ( 7 cases) of the cases extended their nasal tip using the "X-graft," which uses the combination of a caudal septal extension graft and strut graft instead of an ESG and strut graft (Figs. 4, 5).

Treatment for alar retraction was performed in $35 \%$ of all the patients (Table 5, Fig. 6).

\section{Discussion}

With the increase in cosmetic surgery, the incidence of complications or reoperation is also increasing. It is also true that the nasal contracture, which is one of the most severe complications following rhinoplasty, is difficult to treat because it causes

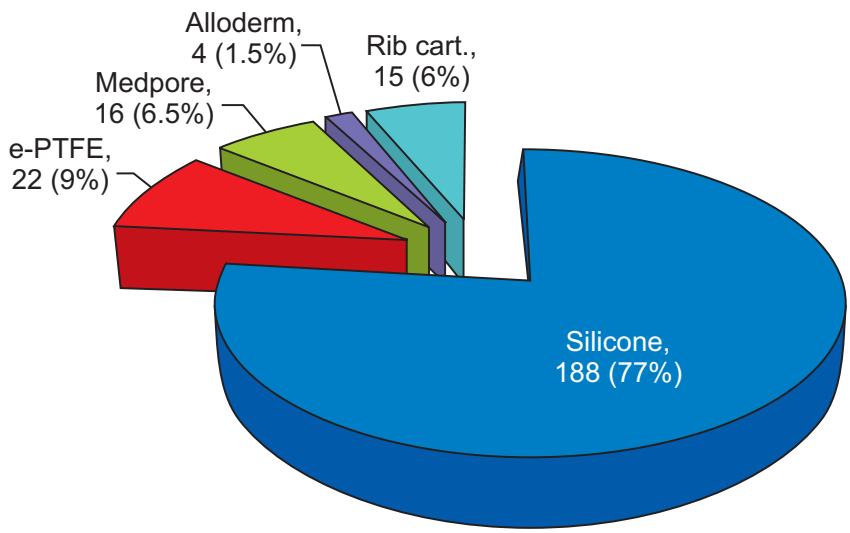

Fig. 3. Previous implant materials.

Table 3. Previous implant materials

\begin{tabular}{llcccccc}
\hline & Type I & Type II & Type III & Type IV & Type V & Type VI & Total \\
\hline Silicone & $3(75 \%)$ & $81(78 \%)$ & $65(79 \%)$ & $19(76 \%)$ & $18(75 \%)$ & $2(33 \%)$ & $188(77 \%)$ \\
e-PTFE & $0(0 \%)$ & $11(10.5 \%)$ & $9(11 \%)$ & $0(0 \%)$ & $2(8.3 \%)$ & $0(0 \%)$ & $22(9 \%)$ \\
Medpore & $1(25 \%)$ & $5(4.8 \%)$ & $4(5 \%)$ & $4(16 \%)$ & $1(4.2 \%)$ & $1(17 \%)$ & $16(6.5 \%)$ \\
Alloderm & $0(0 \%)$ & $2(1.9 \%)$ & $1(1.2 \%)$ & $0(0 \%)$ & $1(4.2 \%)$ & $0(0 \%)$ & $4(1.5 \%)$ \\
Rib cart. & $0(0 \%)$ & $5(4.8 \%)$ & $3(3.8 \%)$ & $2(8 \%)$ & $2(8.3 \%)$ & $3(50 \%)$ & $15(6 \%)$ \\
Total & 4 & 104 & 82 & 25 & 24 & 6 & 245 \\
\hline
\end{tabular}


Table 4. Previous infection history

\begin{tabular}{llcccccc}
\hline & Type I & Type II & Type IIII & Type IV & Type V & Type VI & Total \\
\hline Infection(+) & $4(100 \%)$ & $54(52 \%)$ & $41(50 \%)$ & $23(92 \%)$ & $11(46 \%)$ & $4(66 \%)$ & 137 \\
Infection(-) & $0(0 \%)$ & $50(48 \%)$ & $41(50 \%)$ & $2(8 \%)$ & $13(54 \%)$ & $2(34 \%)$ & 108 \\
Total & 4 & 104 & 82 & 25 & 24 & 6 & 245 \\
\hline
\end{tabular}

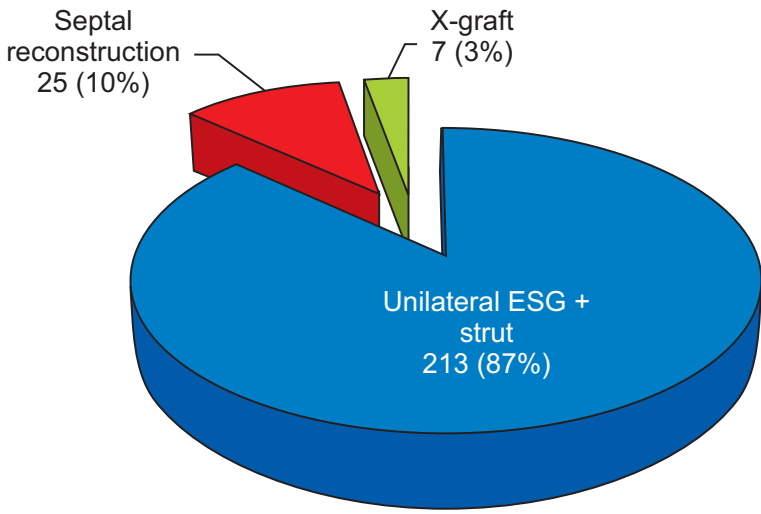

Fig. 4. Nasal tip supporting methods.

a deformity of the soft tissue and supporting structures of the nose, deforming the shape of the nose [2]. The fact that 209 (85\%) of the 245 patients were female does not represent a high incidence of nasal contracture in women. Although the number of cases of men's plastic surgery has increased in the recent years, the rate of rhinoplasty in women is still much higher than that of men; therefore, the rate of side effects is also high and this study is considered to have reflected this phenomenon as well.

Type I (partial type, 1.6\%) is a partial retraction of the skin due to a partial contracture change of the nose. If there is no damage to the epidermis, soft tissue or cartilage can be used to correct it from the underside of the skin (camouflage technique). If there is an accompanying deformity of the epidermis, it is often necessary to perform a correction using flap surgery [3].

Type II (symmetric type, 42.4\%) and type III (asymmetric type, $33.5 \%$ ) were the most common, accounting for $75.9 \%$ of the total. This means that most of the nasal contractures are accompanied by a shortening of the nose tip. In most cases of surgical treatment (90\%), shortening of the nose tip was corrected using nasal ESG (ESG+strut 87\%, X-graft 3\%) [4,5]. Type II (symmetric type) was distinguished from type III (asymmetric type) because in the case of an asymmetric contracture (Type III, asymmetric type), it is often necessary to simultaneously correct the alar retraction.

Approximately $35 \%$ of all subjects had alar retraction or asymmetry of the nostrils that were corrected at the time of the surgical treatment. Mild, moderate, and severe alar retractions were
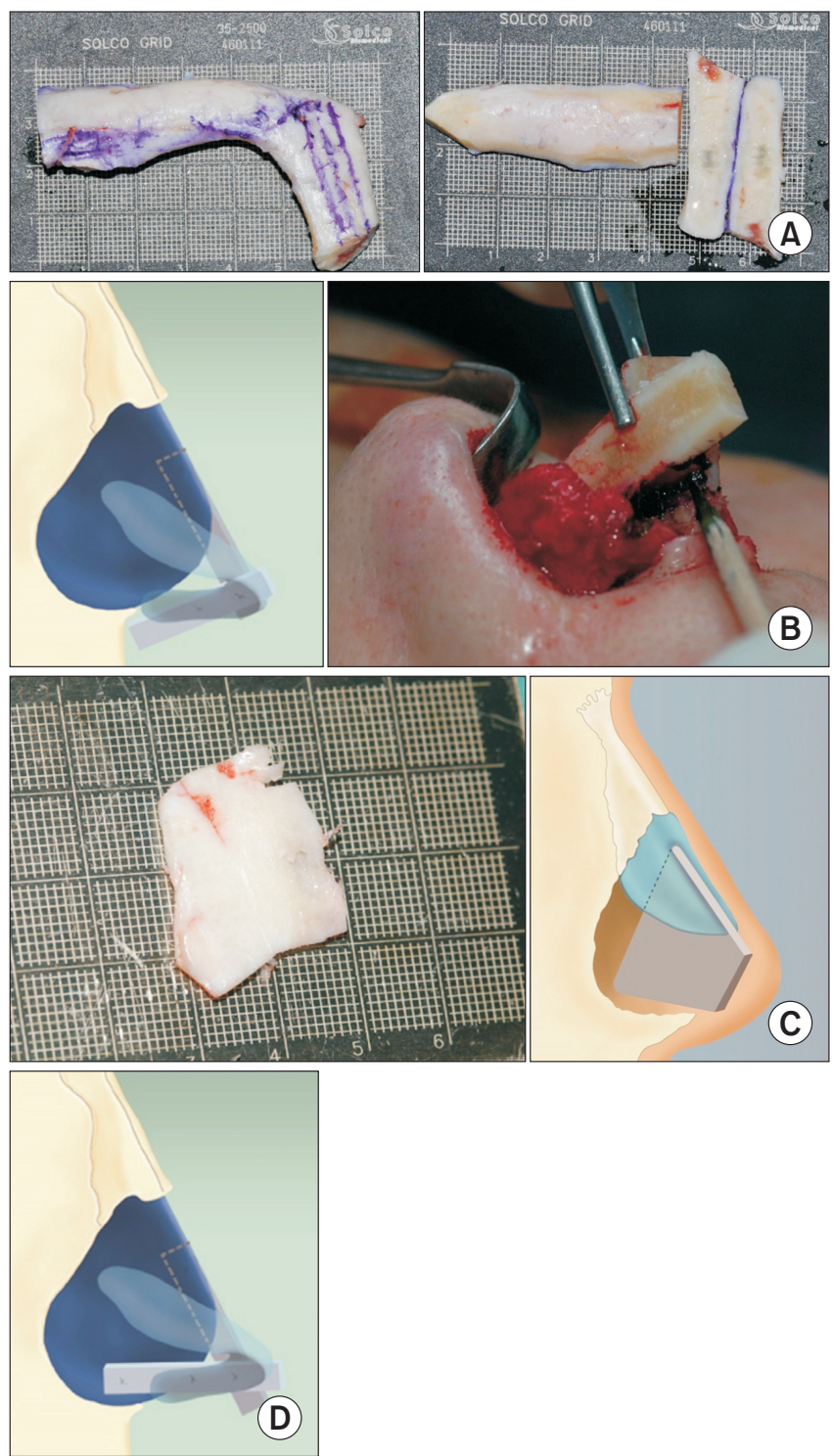

Fig. 5. Tip grafts for contracted nose. (A) Costal cartilage, (B) ESG+strut graft, (C) septal reconstruction, (D) caudal septal extension graft+strut graft (X-graft).

corrected using an alar projection graft, composite graft from the ear, and an alar rotation flap, respectively $[5,6]$. Correction of the asymmetry of the nostrils or alar retraction, as well as extension of the nasal tip is a very important issue in the treatment of nasal contractures. However, correction of an alar retraction 
Table 5. Management of alar retraction

\begin{tabular}{lccccccc}
\hline & Type I & Type II & Type IIII & Type IV & Type V & Type VI & Total \\
\hline Correction(+) & $0(0 \%)$ & $46(44 \%)$ & $24(29 \%)$ & $8(33 \%)$ & $6(25 \%)$ & $2(33 \%)$ & $86(35 \%)$ \\
Correction(-) & $4(100 \%)$ & $58(56 \%)$ & $58(71 \%)$ & $17(67 \%)$ & $18(75 \%)$ & $4(67 \%)$ & $159(65 \%)$ \\
Total & 4 & 104 & 82 & 25 & 24 & 6 & 245 \\
\hline
\end{tabular}
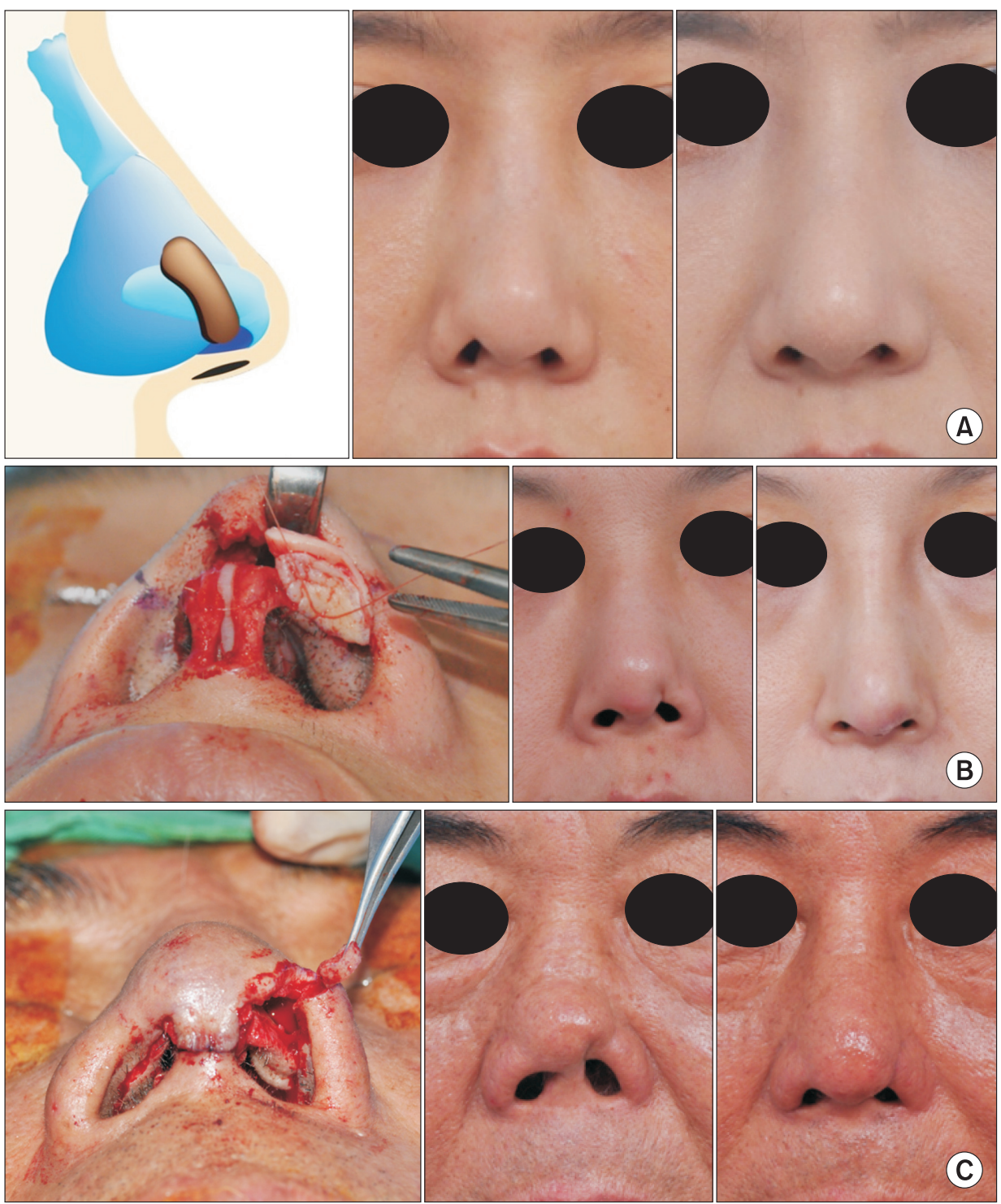

Fig. 6. Treatment of alar retraction. (A) Alar projection graft, (B) composit graft, (C) alar rotation flap.

is not considered to be specifically related to each type of nasal contracture. This should be performed at the discretion of the surgeon, depending on the need for surgical treatment of the nasal contracture. In conclusion, the authors have suggested a new classification system in order to effectively classify and treat nasal contractures. Although silicone implants and inflammation are important factors that are often preceded or accompanied by contractures, additional studies, including histologic examinations, are necessary in order to determine the exact association.

\section{Conflicts of interest}

The authors have nothing to disclose. 


\section{References}

1. Deva AK, Merten S, Chang L. Silicone in nasal augmentation rhinoplasty: a decade of clinical experience. Plast Reconstr Surg 1998;102:1230-7.

2. Jung DH, Moon HJ, Choi SH, Lam SM. Secondary rhinoplasty of the Asian nose: correction of the contracted nose. Aesthetic Plast Surg 2004;28:1-7.

3. Jung DH, Medikeri GS, Chang GU, Hyun SM. Surgical techniques for the correction of postrhinoplasty depressed scars on the nasal tip. JAMA Facial Plast Surg 2015;17:405-12.

4. Jung DH, Loh I. The "X-graft" for nasal tip surgery. Plast Reconstr Surg 2011;128:79e-80e.

5. Jung DH, Tan KS, Jin SG. Nasal tip surgery: nasal lengthening and augmentation in Asian rhinoplasty. J Cosmet Med 2017;1:8-15.

6. Jung DH, Kwak ES, Kim HS. Correction of severe alar retraction with use of a cutaneous alar rotation flap. Plast Reconstr Surg 2009;123:1088-95. 